\title{
Téoros
}

Revue de recherche en tourisme

\section{Les nouveaux territoires du surf dans la ville}

\section{Sylvain Lefebvre et Romain Roult}

Volume 28, numéro 2, 2009

Tourisme, sport et développement

URI : https://id.erudit.org/iderudit/1024807ar

DOI : https://doi.org/10.7202/1024807ar

Aller au sommaire du numéro

Éditeur(s)

Université du Québec à Montréal

ISSN

0712-8657 (imprimé)

1923-2705 (numérique)

Découvrir la revue

Citer cet article

Lefebvre, S. \& Roult, R. (2009). Les nouveaux territoires du surf dans la ville. Téoros, 28(2), 55-62. https://doi.org/10.7202/1024807ar

\section{Résumé de l'article}

La culture surf qui a tant marqué la côte ouest américaine à partir des années 1950, culture qui s'est mondialisée et commercialisée avec plusieurs tendances toutes aussi variées et inusitées les unes que les autres, a créé un univers aux proportions imposantes dans les dix dernières années. L'industrie du divertissement, du sport et du loisir est au centre de cette révolution, mais aussi celle du vêtement, de la mode et d'autres produits dérivés. Plus significatif encore, le surf original a influencé plusieurs pratiques sportives tant sur l'eau que sur terre ou sur neige, et désormais les intervenants touristiques de même que ceux en aménagement du territoire doivent s'adapter et composer avec ces nouvelles réalités. L'article dressera un panorama de cette constellation d'activités, qui ont le surf comme point de départ, et de leurs évolutions récentes. Puis, plus spécifiquement, il sera question des impacts de ces activités sur l'espace urbain à travers des modes d'appropriation de territoires en apparence plutôt inadaptés aux « sports de glisse ».
Ce document est protégé par la loi sur le droit d'auteur. L'utilisation des services d'Érudit (y compris la reproduction) est assujettie à sa politique d'utilisation que vous pouvez consulter en ligne.

https://apropos.erudit.org/fr/usagers/politique-dutilisation/ 


\title{
Les nouveaux territoires du surf dans la ville
}

\author{
Sylvain LEFEBVRE \\ Professeur \\ Département de Géographie \\ Université du Québec à Montréal \\ lefebvre.sylvain@uqam.ca \\ Romain ROULT \\ Doctorant \\ Département d'études urbaines et touristiques \\ Université du Québec à Montréal \\ roult.romain@courrier.uqam.ca
}

RÉSUMÉ: La culture surf qui a tant marqué la côte ouest américaine à partir des années 1950, culture qui s'est mondialisée et commercialisée avec plusieurs tendances toutes aussi variées et inusitées les unes que les autres, a créé un univers aux proportions imposantes dans les dix dernières années. L'industrie du divertissement, du sport et du loisir est au centre de cette révolution, mais aussi celle du vêtement, de la mode et d'autres produits dérivés. Plus significatif encore, le surf original a influencé plusieurs pratiques sportives tant sur l'eau que sur terre ou sur neige, et désormais les intervenants touristiques de même que ceux en aménagement du territoire doivent s'adapter et composer avec ces nouvelles réalités. L'article dressera un panorama de cette constellation d'activités, qui ont le surf comme point de départ, et de leurs évolutions récentes. Puis, plus spécifiquement, il sera question des impacts de ces activités sur l'espace urbain à travers des modes d'appropriation de territoires en apparence plutôt inadaptés aux «sports de glisse».

Mots-clés: Surf, skateboard, longboard, sports extrêmes, espace urbain, culture, glisse, modes de vie, Montréal.

Depuis plusieurs années, la culture surf des années 1950 fait un retour spectaculaire dans les modes de consommation et les pratiques sportives et récréatives urbaines. Mondialisation d'un style de vie qui prône la liberté, l'esprit de la glisse, l'individualité et le fun, cette culture surf est désormais omniprésente dans les médias, l'industrie du vêtement, celle du divertissement et du loisir (De Soultrait, 1995; Kampion et Brown, 1998; Poirier, 2000). Toute une nouvelle génération d'activités sportives a émergé de cette tendance lourde : les lifestyle sports (Wheaton, 2004), des sports auxquels les individus et quelques groupes restreints s'identifient avec force par des symboles et des référents qui débordent sur le mode de vie plus général du pratiquant. Il n'y a pas de traduction satisfaisante pour lifestyle sports. Nous utiliserons par défaut l'expression "sports - mode de vie», un ensemble de pratiques, de modes de consommation et de rites qui représentent un véritable phénomène social. Ce phénomène influence surtout plusieurs pratiques ludiques et sportives tout en mettant en évidence de nouveaux modes d'appropriation territoriale.

Les travaux du GREF (Groupe de recherche sur les espaces festifs) du département de géographie de l'UQAM (Université du Québec à Montréal), portant notamment sur les sports de glisse, ont permis de documenter plusieurs aspects de ces sports - mode de vie depuis une dizaine d'années (Wieland, 1999; Lefebvre et Latouche, 2001; Lefebvre et Chouinard, 2003; Filiatrault, 2006). Le phénomène du skateboard, celui du patin à roues alignées et la pratique du longboard ont été les principaux objets d'étude. Plusieurs observations sur le terrain et quelques entretiens avec des intervenants dans le domaine de ces pratiques sportives et de l'industrie des équipements sportifs ont permis de bonifier l'ensemble de nos résultats.

À partir d'une revue de littérature, cet article propose de tracer les grandes étapes de l'évolution des sports - mode de vie, dans le contexte du milieu urbain.

\section{Les fondements de la culture surf}

Depuis déjà nombre d'années, il y a diversification, multiplication et hybridation de plusieurs pratiques sportives qu'elles soient traditionnelles ou non. Dans ce foisonnement de produits, d'activités nouvelles et de nouveaux modes d'appropriation des équipements et espaces sportifs, la culture surf, celle héritée de la côte ouest américaine des années 1950, s'est propagée et renouvelée de façon spectaculaire (PoizatNewcomb, 1999; Dolnicar et Fluker, 2003). Originaire de 


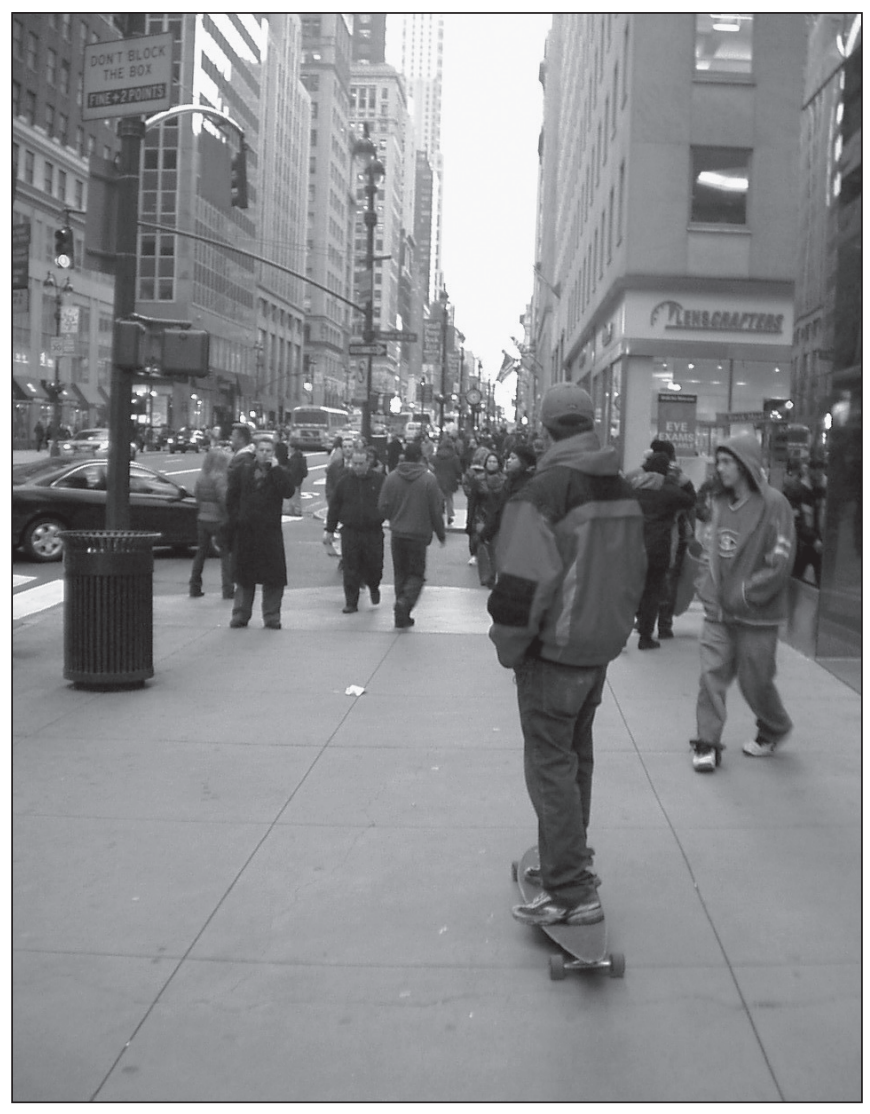

ILLUSTRATION 1 : Pratique du longboard dans les rues de Manhattan (photo : Sylvain Lefebvre).

plusieurs îles du Pacifique, la pratique du surf fut découverte par des missionnaires au XIX ${ }^{e}$ siècle et semblait déjà associée à un mode de vie spirituelle pour des milliers d'habitants (Bessas, 1982; Poirier, 2000). Loin d'être un simple loisir, le surf était pratiqué par la royauté de plusieurs de ces îles et se définissait comme une expérience profonde, noble et en harmonie avec les éléments de la nature. Mise au rancart par les instances religieuses colonisatrices, qui y voyaient une activité inutile, trop festive et dommageable moralement, le surf fut réintroduit seulement au début du $\mathrm{XX}^{\mathrm{e}}$ siècle à Hawaii, en Australie et en Californie (Kampion et Brown, 1998). Le retour aux valeurs ancestrales du surf (expérience intérieure et spirituelle, respect de l'environnement naturel, recherche du plaisir) fut privilégié par certaines figures emblématiques de ce sport et est demeuré depuis (Lhote, 2003). Ce sont surtout les années 1950 et 1960 qui ont popularisé ce sport à un large public par des productions hollywoodiennes (Gidget, The Endless Summer, Ride the Wild Surf) et des groupes musicaux (surf rock) ayant une grande visibilité internationale (Beach Boys, Ventures, Dick Dale) (Kampion et Brown, 1998; Colburn, 2002). Les années 1960 et 1970 ont toutefois figé la réputation des surfeurs autour de leurs valeurs rebelles, anticonformistes, de leurs habitudes de vie marginales et de l'utilisation de drogues. La culture surf amorça un virage radical vers la fin des années 1970 avec la professionnalisation du sport, créant ainsi un clivage important entre les purs et durs (les Soul Surfers) et les athlètes récupérés par l'industrie naissante dans le secteur de l'équipement et des vêtements (Kampion et Brown, 1998; Poirier, 2000).

Aujourd'hui, la culture surf est médiatisée et publicisée massivement à travers des produits et dérivés qui totalisaient, en 1997, des revenus de plus de sept milliards de dollars US par année (Brown, 1997). Les entreprises Rip Curl, Quiksilver et Billabong, pour n'en nommer que quelques-unes, sont présentes dans plus de 70 pays et c'est sans compter le nombre impressionnant d'entreprises et de multinationales qui utilisent des images de surf pour promouvoir leurs propres produits (Coca-Cola, Motorola, etc.). À la fin des années 1980, le nombre d'adeptes de surf était déjà estimé à 17 millions de pratiquants réguliers à travers le monde (Brown, 1997). On imagine bien ce que peut représenter dans ce contexte le nombre de personnes s'identifiant plus globalement à la culture surf sans nécessairement pratiquer le sport en question.

La surf a généré et inspiré plusieurs autres activités sportives de glisse. Les deux principaux dérivés du surf de vague qui se sont maintenus et qui se sont généralisés massivement sont le skateboard (planche de rouli-roulant ou planche à roulettes) et le snowboard (planche à neige) (Borden, 2001). En 2005 , on comptait près de 11 millions de skateboardeurs aux États-Unis et cinq millions de snowboardeurs (SGMA, 2006). Nous adoptons ici la francisation généralement utilisée dans la littérature francophone pour traduire surfer, snowboarder, longboarder, etc., pour surfeur, snowboardeur et longboardeur, etc. L'engouement pour ces deux sports n'a fait qu'augmenter au fil des années et, un peu à l'image du surf, il y a une professionnalisation et une commercialisation très significatives de ces deux pratiques avec, pour ses adeptes, les mêmes symboles, référents identitaires et clivages dans les types de pratique (Ford et Brown, 2006).

\section{Culture surf et sports de glisse}

Il est plutôt difficile de différencier avec précision les sports alternatifs des activités sportives traditionnelles. On qualifie une activité de «sportive» lorsqu'elle regroupe simultanément trois composantes : une dimension ludique, une dimension compétitive (contre soi, autrui ou un groupe de personnes) et finalement un effort physique important (Augustin, 1994 : 15-16). Les sports traditionnels organisés ou non contiennent ces trois éléments. Or, dans les trois dernières décennies, une nouvelle gamme d'activités qualifiées aussi de sportives est apparue, mais avec des labels les distinguant des pratiques socialement et culturellement reconnues, bien établies et parfois institutionnalisées. Sports extrêmes, sports alternatifs, sports post-modernes, sports - mode de vie sont autant de dénominateurs appliqués à une gamme très diversifiée et très complexe de pratiques sportives moins populaires, distinctes des sports traditionnels et qui se démarquent par leur caractère de contre-culture, non-intégré à une culture sportive dominante, soucieuses de créer une image distincte, parfois rebelle et liée à un besoin d'altérité identitaire (Pégard, 1998; Karsten et Pel, 2000). Sur ce dernier point, il y a véritablement un processus d'individuation à travers ces pratiques sportives, très souvent à la marge des processus et des lieux traditionnels 
de socialisation. Jeux sportifs, nouvelles expériences de plein air, redécouverte de la nature ou d'un environnement banalisé (la trame urbaine), les sports de glisse transforment les valeurs individuelles et les territorialités vécues par ses adeptes (Augustin, 1994; Vivoni, 2009). Dans cette perspective, on assiste à la consolidation d'un nouveau paradigme dans l'évolution du sport, celui d'un affranchissement du cadre référentiel traditionnel du sport institutionnalisé par certains profils types de pratiquants (Filiatrault, 2006).

L'«esprit glisse» a historiquement été créé de toutes pièces par des groupes de surfeurs de certaines plages réputées pour leurs vagues (Bessas, 1982; Ford et Brown, 2006). En opposition au conformisme de la culture américaine dominante de l'époque et des Trente Glorieuses, un savant mélange de valeurs contestataires, de sympathie pour la Beat Generation et de tribalisme identitaire devait fonder cette culture surf qui fut rapidement récupérée, mise sur le marché et popularisée à toute la société nord-américaine (Carol, 1995; Poirier, 2000). Cette culture surf s'est appuyée d'abord sur un mode de vie marginal, rebelle, donnant priorité au ludique et aux sensations extrêmes de l'activité physique elle-même (glisse de la vague, fluidité, style, acrobaties). Certains auteurs ajoutent même cette idée de "déguisement», importante aux communautés de surfeurs en quête de distinctif et de marquage dans l'espace de leur style de vie si particulier (Loret, 1995; Pégard, 1998; Hamm, 2004). Les mouvements contestataires des années 1960 sont venus renforcer les bases de cette culture surf avec leurs corollaires et dérivés dans le domaine des arts, de la culture et de la musique (Colburn, 2002).

On comprendra ici que la culture surf déborde largement des activités physiques de glisse qui lui sont associées. Yves Bessas (1982) donne le nom de «glisse» à un système de valeurs fondé sur des sensations nouvelles personnelles, libérées de toute contrainte (prendre son pied, s'éclater), qui prennent appui sur un mouvement fluide (la glisse) souvent hors-piste, avec une recherche du plaisir de l'absurde et l'oubli de la raison. "À chacun sa passion puisque les paysages sont nés pour qu'on les glisse» (Bessas, 1982 cité dans Augustin, 1994 : 25). La planche à voile, la planche à roulettes, le parapente, la planche à neige, le surf de rue (longboard), le surf de rivière, l'escalade, le kayak de rivière ou de mer, le parkour, pour ne citer que ces quelques activités de glisse, ont toutes la particularité de s'approprier les paysages, d'en explorer de nouveaux, de marquer ces nouveaux territoires en glissant à travers eux (Lefebvre et Chouinard, 2003). À noter que le parkour est une activité de glisse extrême dans la jungle urbaine qui consiste à exécuter avec le corps uniquement des déplacements et des figures acrobatiques pour contourner et utiliser toutes les possibilités du mobilier urbain, des façades et de l'architecture. De même, il n'y a pas de traduction satisfaisante pour longboard; cette planche à roulette est généralement beaucoup plus longue que sa petite cousine et reste beaucoup plus facile à manœuvrer.

Les labels fun, cool, hip, slick et de nombreux autres de même acabit sont généralement associés à toute une symbolique et à une mythologie entourant l'objet culte qu'est devenue la planche de surf. Dans l'imaginaire collectif, le surfeur rebelle des années 1950 s'est adapté aux courants dominants de la mondialisation du sport, de la démocratisation des sports de glisse et de la marchandisation d'une certaine forme d'individualisme. Plus acceptable socialement, le surfeur est désormais amadoué, apparaît moins rétif et peut ainsi devenir une référence intéressante pour les jeunes et les moins jeunes en quête de singularité (Borden, 2001). Dans l'univers complexe et omniprésent de cette culture surf, le véritable surfeur ou skateur refusant toute forme de récupération commerciale et capitalisant souvent sur cette image radicale pour ses propres fins, côtoie une multitude d'individus habillés avec des vêtements griffés par des entreprises de surf, de skateboard ou autres sports de glisse, ces mêmes individus parfois avec une planche sous le bras sans avoir glissé une seule fois sur une vague, la neige ou le bitume (Arthur, 2003; Humphreys, 2003).

Arthur (2003: 162, traduction libre) observe que « [1] e surf est un phénomène qui intéresse une quantité phénoménale de consommateurs et ce, bien au-delà du nombre de surfeurs qui représentent l'objet central du marketing. Il y a même une boutique de surf très connue à Singapour mais les eaux les plus tumultueuses qu'on retrouve dans cette petite ville-état du Sud-Est asiatique sont les gouttes de condensation sur les fenêtres de la boutique en question. De même, on retrouve une boutique de surf directement située entre Chanel et Prada en plein Manhattan, dans le centre-ville de New York». L'engouement pour la culture surf prend ainsi des formes inusitées, parfois même un peu caricaturales. Ce n'est plus tant l'acte de surfer qui est au centre de cette culture, mais plutôt les représentations collectives, symboliques et identitaires d'une attitude et de valeurs bien précises que l'on associe généralement aux sports de glisse (Wieland 1999; Sayeux, 2008).

Ces sports - mode de vie réintroduisent la dimension ludique (le fun) qui avait été écartée ou minimisée dans l'univers du sport réglementé et institutionnalisé depuis le XIXe siècle. Cette tendance est à ce point lourde que même l'univers des sports plus traditionnels se retrouve transformé par un retour progressif du facteur festif et ludique. En effet, les championnats et autres grands événements sportifs classiques redécouvrent les vertus festives, ludiques, familiales et carnavalesques du spectacle sportif, et de la participation libre et spontanée. Le championnat de football européen qui se déroulait en Autriche et en Suisse (Euro 2008) a été un excellent prétexte au même moment, à Montréal, pour organiser plusieurs activités de plein air célébrant ce sport : bien sûr visionner les matchs sur écrans géants dans les rues et les parcs, mais surtout une foule d'activités festives complémentaires (festivals sportifs et cinématographiques sur le thème du football, ateliers pour familles, cours d'initiation, expositions thématiques sur le football et ses dérivés, etc.).

Les sports de glisse s'appuient sur la consommation d'objets qui ont pour point de départ la planche (le board), mais des objets qui se transforment de façon parfois spectaculaire. Ce sont des produits peu connus, inusités et innovateurs, utilisant des technologies de pointe et de nouveaux matériaux, toujours à la marge des produits plus connus, mais surtout en constante évolution et en démarcation par rapport aux produits de même catégorie. Ce faisant, des produits hybrides sont créés, dans un formidable foisonnement, et génèrent à eux seuls de nouvelles activités et de nouvelles pratiques. 


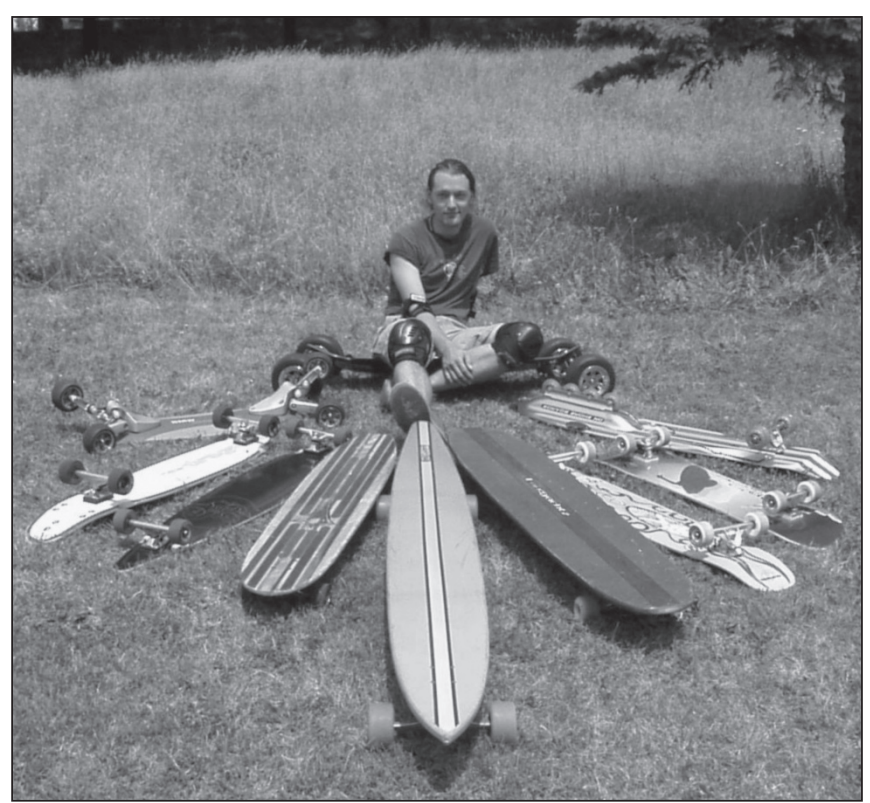

ILLUSTRATION 2 : Un pratiquant de longboard avec quelques modèles de planche très différents (photo : Sylvain Lefebvre).

Ainsi, les activités «sportives - mode de vie», alimentées par ces innovations rapides, représentent un phénomène de dispersion technologique et d'hybridation de produits. Ces activités échappent ainsi aux catégorisations, typologies et autres classifications qui permettraient notamment de normaliser des lieux ou des espaces adaptés à leurs besoins.

En émergence progressive ces quatre ou cinq dernières décennies, les sports - mode de vie et les sports de glisse ont évolué au point de s'incruster de façon plus solide dans le paysage ludosportif. On l'a vu, les pratiques sportives de glisse utilisant diverses formes de planches ont d'ailleurs récupéré et conservé des références culturelles très puissantes de la culture surf californienne des années 1950 : liberté individuelle, harmonie avec les éléments naturels, expérience spirituelle intérieure, défis individuels pour soi et affirmation identitaire. Leur caractère, souvent qualifié à tort "d'extrême", s'est davantage mué en une culture beaucoup moins rebelle, marginale ou contestataire pour faire appel aux valeurs communautaires, participatives et récréatives, les rendant ainsi plus socialement acceptables, mais aussi plus accessibles (Loret, 1995; Adamkiewicz, 2001).

Au Canada, le nombre d'adeptes dans les sports de glisse est en hausse constante (CCS, 2007). L'industrie de la planche à neige prévoyait qu'en 2004-2005, plus de $50 \%$ des usagers des pistes de ski en Amérique du Nord seraient des planchistes réguliers. Au Canada, c'est plus d'un million et demi de snowboardeurs qui fréquentent les pentes de ski, une clientèle qui représente plus de $30 \%$ des pratiquants de tous les sports d'hiver de glisse confondus alors qu'aux États-Unis, ils représentent près de cinq millions d'adeptes (CCS, 2007; NSSRA, 2008). En France, la planche à neige compte aussi plus de cinq millions de pratiquants (ODIT France, 2009). La planche à roulettes compte près de 11 millions de pratiquants aux États-
Unis en 2005, une augmentation de $55 \%$ en seulement dix ans (SGMA, 2006). Ce marché s'est stabilisé en Amérique du Nord et désormais la pratique du longboard vient cannibaliser une part du marché du skateboard classique, son cousin plus petit et moins maniable. L'évolution de la pratique du longboard est plutôt rapide dans les zones urbaines et malgré l'absence de données précises, certaines estimations de l'industrie chiffrent à un intervalle de $15-25 \%$ le taux de croissance annuel des ventes de planches spécifiques à cette activité. Des enquêtes ont été réalisées en 2004 et 2005 sur le profil socio-économique des longboardeurs de Montréal au département de géographie de l'UQAM de même que des entretiens auprès de détaillants spécialisés montréalais (Filiatrault, 2006). Les résultats sont intéressants dans la mesure où le taux de croissance du nombre de pratiquants semble très élevé et que le profil type de ces derniers fait ressortir clairement une tranche d'âge moyenne de 25-35 ans, avec un niveau de scolarité et de revenu plutôt élevé. L’image caricaturale du jeune délinquant (15-20 ans) avec son skateboard et ses comportements déviants est non seulement une aberration dans les études empiriques sur le sujet, mais, surtout, ne colle vraiment pas à la pratique émergente du longboard (Wieland, 1999; Filiatrault, 2006).

\section{Rupture et nouvelles expressions sportives}

Pratiquer des sports de "planche» représente pour plusieurs de ses adeptes, une forme de liberté et de créativité dans l'univers parfois envahissant de la performance physique, de la compétition et des normes ou contraintes qui régissent certaines activités sportives. Surfer une vague, la neige ou la rue c'est surtout se déplacer, moyennant un effort physique bien sûr, mais dans un mode déambulatoire, flexible, libre au niveau des mouvements ou des trajectoires empruntées. La sensation prend le dessus sur la prestation. L'expérience et les plaisirs générés supplantent les résultats et les records mesurés.

Tout l'intérêt des sports de glisse depuis l'apparition de la culture surf réside dans de nouveaux «rapports au corps, d'autres rapports à l'autre, d'autres rapports à l'environnement, bien différents de ceux qui ont été historiquement prônés par le sport olympique. En réalité, la glisse apparaît comme un processus d'évolution à la fois culturelle et technique.» (Loret et Waser, 2001 : 17) Les valeurs dominantes véhiculées par la glisse confrontent certains stéréotypes sur le sport lui-même telles la concurrence, la justice et l'égalité des chances. Qu'ils soient qualifiés d'extrêmes ou d'alternatifs, les sports de glisse se caractérisent plutôt par une hiérarchisation acceptée des pratiquants, la libre créativité dans les styles de pratique de même que la connivence/complicité entre pratiquants (Loret et Waser, 2001). Glisser dans le paysage montagneux (l'escalade libre sur des parois, les descentes de luge de rue), le paysage nautique (vagues de mer et de rivières, vagues provoquées par des embarcations spécialisées) ou le paysage urbain (la rue, le mobilier, les espaces publics), c'est d'abord découvrir et s'approprier des sites inattendus, atypiques avec des références nouvelles à des sous-cultures artistiques, et plus globalement à des modes de vie particuliers. La glisse «s'inscrit pleinement dans une mouvance sociale qui valorise l'instantanéité, le libre accès, l'absence de structures hiérarchiques et l'autorégulation.» (Loret et Waser, $2001:$ 17-18). 


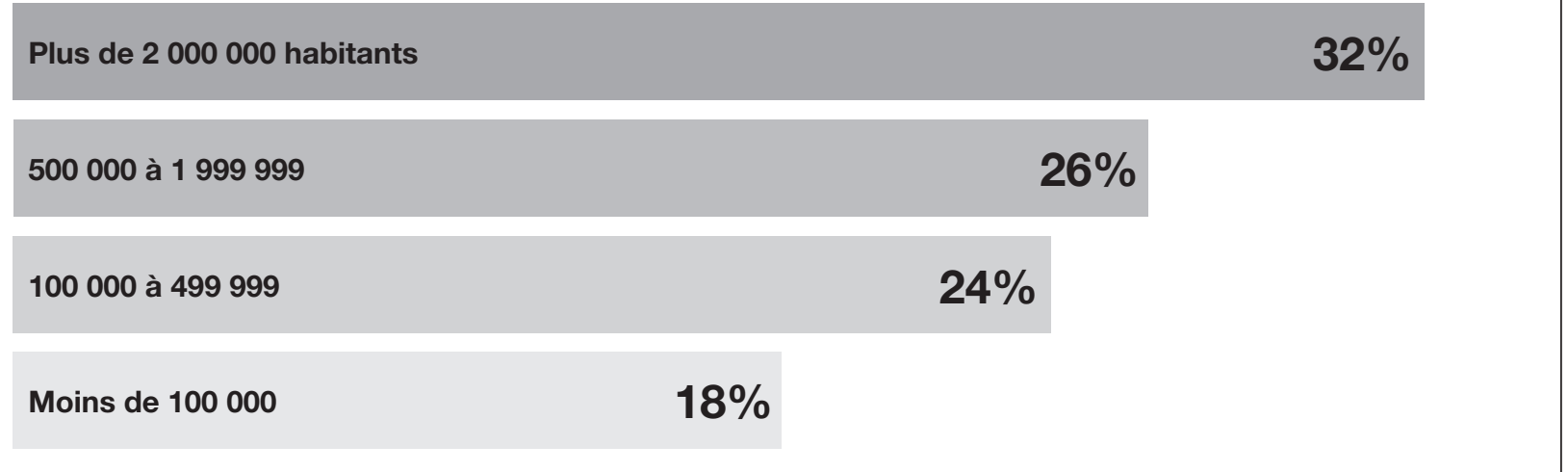

ILLUSTRATION 3 : Distribution de pratiquants de la planche à roulettes selon la taille des villes - États-Unis, 2005

(source : Données - Skateboard USA (SGMA 2005)).

«La dimension extrême de ce sport n'est pas indispensable. Il y a beaucoup de personnes qui, vous savez, font de la planche à neige de façon plutôt conservatrice. Ce qui me semble plus intéressant, c'est que cette activité est devenue un sport - mode de vie (...) Et je ne suis pas sûr pourquoi, mais il y a quelque chose d'unique dans les sports de planche.» (Trad. libre des propos $\mathrm{du}$ fondateur d'une marque très connue de planches à neige, Jake Burton cité dans Wheaton, $2004: 4)$

Si les activités propres à la culture surf et les sports de glisse s'expriment à travers la spontanéité, le goût de la provocation, la nouveauté et par l'individuation, elles ont surtout un potentiel de singularisation, de distinction et d'affirmation de ses pratiquants (Filiatrault, 2006). La planche, ou le board, est un objet emblématique que l'on utilise pour glisser, mais que l'on peut aussi emporter sous le bras et qui, pour plusieurs adeptes, représente un symbole identitaire fort (Ford et Brown, 2006). Mises en scène variables du corps dans le déplacement, mais aussi mises en scène atypiques du paraître et du statut de surfeur dans l'espace urbain. La publicité et les médias en général l'ont très bien compris et utilisent avec abondance les planches de surf, longboards et autres dans plusieurs situations toutes aussi opportunistes les unes que les autres. La planche comme objet cool permet ainsi de rejoindre des clientèles plus jeunes, branchées, et vient surtout se rallier au style de vie véhiculé par la culture surf.

\section{Les territoires de la glisse : le cas montréalais}

L'espace urbain est très sollicité par les sports de glisse. Rues, espaces publics, semi-publics ou privés, parcs, pistes cyclables, mobilier urbain, la ville apparaît comme un lieu de prédilection permettant des comportements de glisse inédits. Non pas qu'il y ait ici détournement des usages courants des espaces urbains au profit de nouvelles pratiques sportives, mais plutôt un enrichissement et de nouvelles qualifications de certains lieux et de certains territoires (Adamkiewicz, 2001). Plusieurs auteurs (Loret, 1995; Adamkiewicz, 2001; Lefebvre et Latouche, 2001) intéressés par la question reconnaissent ainsi que les sports de glisse ne peuvent être perçus comme des nuisances, des déviances ou des anomalies dans la ville, mais comme des réappropriations de l'espace public, des revendications de l'urbain comme lieu de l'expression sociale (Pégard, 1998; Wieland, 1999; Adamkiewicz, 2001; Vivoni, 2009).

Dans un contexte où il existe une demande sociale croissante pour des modes de déplacement urbain "propres» ou non motorisés, ces nouveaux déplacements de glisse ont un capital de sympathie auprès des populations et bénéficient d'une certaine tolérance des instances publiques, et ce, malgré les défis non résolus de la cohabitation avec les autres modes de transport et l'absence d'espace urbain spécifique et adapté à chacune des nouvelles pratiques de glisse (Lefebvre, 2004).

Certaines enquêtes démontrent clairement que la ville est devenue un espace privilégié pour la glisse urbaine (Lefebvre et Latouche, 2001; SGMA, 2006). $82 \%$ des quelques 11 millions de skateboardeurs aux États-Unis pratiquent dans des villes d'au moins 100000 habitants et $32 \%$ dans les villes de plus de deux millions d'habitants (illustration 3) (SGMA, 2006). Véritable phénomène métropolitain, la glisse urbaine auparavant chasse gardée des cyclistes et des patineurs à roues alignées doit désormais composer avec un nombre croissant de skateurs et de longboardeurs.

Dans les faits, pour certaines pratiques comme le longboard, ce n'est pas tant l'aménagement de nouveaux équipements ou l'adaptabilité de certains espaces urbains qui posent problème, mais plutôt la permissivité de leur usage dans la rue et le cadre urbain existant. Les nouveaux usages de glisse urbaine nécessitent de préférence un encadrement cohérent au niveau des techniques de déplacement et des manœuvres sécuritaires pour les autres usagers de la ville (limite de vitesse, respect du code de la sécurité routière, codes de navigation en cohabitation avec d'autres types de déplacement, etc.). Plus globalement, les sports de glisse bousculent nos représentations de l'usage de la ville, mais aussi certaines techniques et certains processus relevant de l'urbanisme et de l'aménagement du territoire (Lefebvre et Latouche, 2001). En effet, l'espace public, ou du moins certains lieux urbains, doivent être repensés, adaptés et aménagés pour accommoder la glisse. Choix des matériaux, zones et couloirs réservés, gabarits et formes du cadre bâti, voilà autant d'éléments qui doivent désormais 


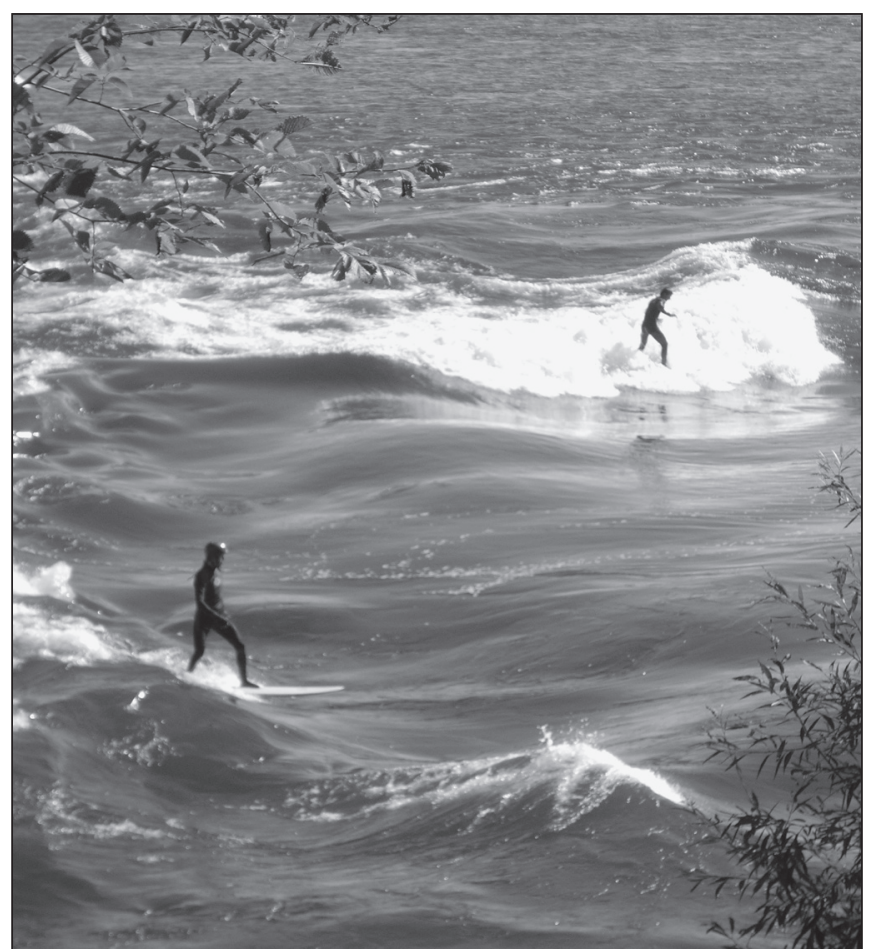

ILLUSTRATION 4 : Surf de rivière sur le fleuve Saint-Laurent à Montréal près de la pointe du Havre (photo : Sylvain Lefebvre).

être pris en compte dans cette superposition des usages et des déplacements (Borden, 2001; Vivoni 2009).

Montréal est une ville qui a été frappée de plein fouet par le phénomène de la culture surf et de la glisse en général. Ville reconnue pour ses indices de qualité de vie élevés, pour sa vocation festive et ludique de réputation internationale (festivals, sports événementiels de prestige, arts et culture, tourisme urbain, etc.), elle a su mettre de l'avant plusieurs espaces et lieux de pratique de glisse urbaine. À la base, le vélo fut probablement à l'origine d'un des plus importants réseaux urbains de pistes cyclables en Amérique du Nord. La croissance des déplacements récréatifs et utilitaires en vélo a été systématique et s'est accompagnée d'une consolidation des pistes et des voies cyclables dans la ville. Par la suite, dès le début des années 1980, les adeptes du patin à roues alignées ont pris d'assaut ce réseau cyclable de même que les rues de la métropole. La cohabitation entre ces deux modes de déplacement n'a pas été sans créer des conflits d'usage du réseau cyclable (Lefebvre et Latouche, 2001). Néanmoins, la montée en puissance du phénomène du patin à roues alignées a témoigné de la réappropriation de la rue et des espaces publics par une clientèle de glisse urbaine nouvelle, au profil socio-économique variable et motivé par une expérience motrice pratique et ludique. La pratique de la planche à roulettes, quant à elle, est plutôt mal desservie par l'offre en équipements et lieux de pratique. Aux États-Unis seulement, les quelques 11 millions de skateboardeurs ont moins de 3000 parcs spécialisés pour ces planches, alors que la demande réelle est estimée à plus de 50000 parcs (SGMA, 2006; Vivoni, 2009). Cette situation fait en sorte que plus de

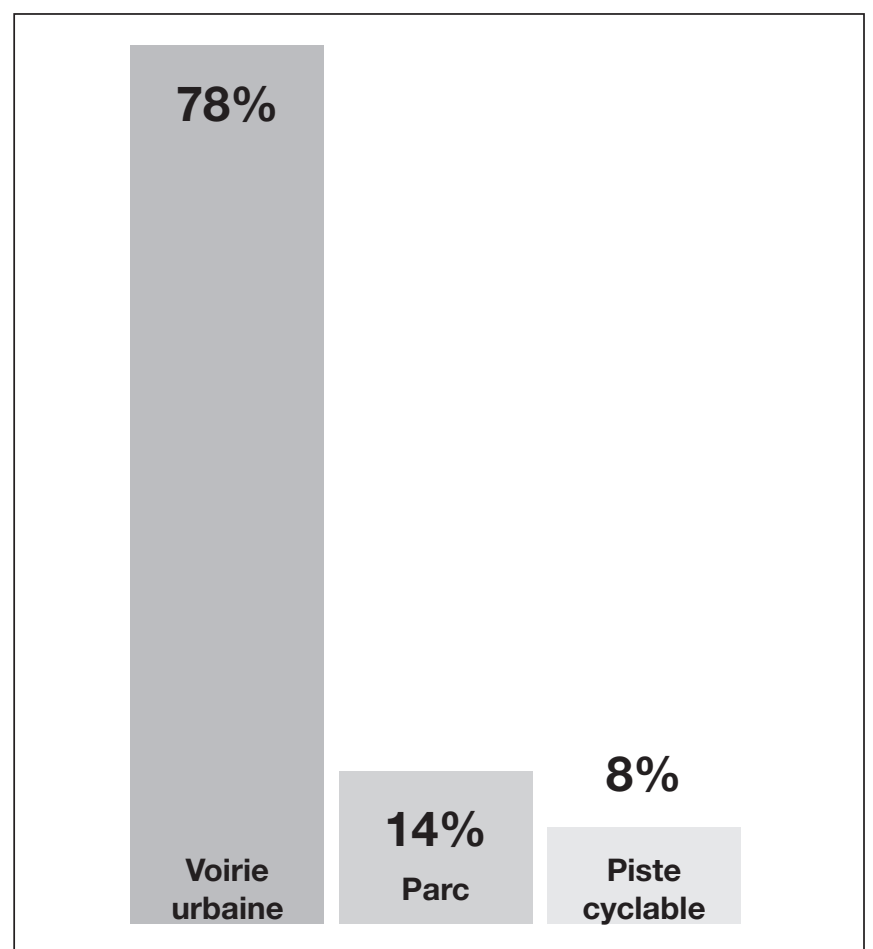

ILLUSTRATION 5 : Lieu de pratique du longboard - Montréal, 2004 (source : Données - Filiatrault 2004).

$80 \%$ des pratiquants de planche à roulettes utilisent la rue et les espaces publics (pratique communément dénommée street skating) (Borden, 2001; Hamm, 2004). Montréal n'échappe pas à ce phénomène avec seulement 32 parcs disséminés sur le territoire de l'île.

Depuis, avec un retour et une stabilisation de la pratique de la planche à roulettes, alimentés par une tolérance variable de leur présence sur la voie publique, une nouvelle glisse urbaine s'est insérée graduellement dans le réseau cyclable et les rues montréalaises : une longue planche à roulettes communément appelée longboard. Encore une fois, il n'y a pas de traduction satisfaisante pour le mot longboard. À l'origine, un longboard était, et représente toujours, une très longue planche de surf (environ trois mètres) qui permettait de surfer les plus petites vagues. En essayant de reproduire le surf sur l'asphalte, des planches à roulettes plutôt longues ont été conçues et ont emprunté le terme qui est demeuré depuis. Ces longboards ont des longueurs de $80 \mathrm{~cm}$ et plus, pouvant même atteindre parfois plus de deux mètres! Ils sont redevenus particulièrement populaires à l'échelle internationale dans les dix dernières années (Filiatrault, 2006).

La pratique du longboard à Montréal est désormais inscrite dans le paysage urbain. On peut désormais surfer la rue avec ces longues planches à roulettes qui se distinguent passablement de leurs cousines plus petites. Plus longues, maniables, stables, plus performantes aussi, ces planches sont utilisées comme de véritables planches de surf, les sensations et les mouvements pouvant être reproduits de façon variable selon les modèles. Elles représentent un heureux compromis entre 
les petites planches à roulettes plus radicales et moins fluides et la possibilité de rouler avec aisance et un niveau d'habileté technique raisonnable. Les longboards ne sont pas utilisés dans les skateparks (parcs aménagés pour les petites planches à roulettes et qui desservent une pratique acrobatique sur des modules spécifiques). Il n'existe d'ailleurs aucune consigne réglementaire ni aucune forme d'accommodement pour leur utilisation. Dans une majorité de villes nord-américaines, elles sont donc tolérées sur la voie publique, dans les parcs et sur les pistes cyclables. Faciles à utiliser pour la promenade, les longboards laissent des traces concrètes de la culture surf et de l'esprit de la glisse dans l'espace urbain (illustration 5) (Filiatrault, 2006). Un peu à l'image des premiers balbutiements des patins à roues alignées, la publicité, l'industrie du vêtement et certaines compétitions sportives confirment désormais que la pratique du longboard n'est pas près de disparaître.

Dans le contexte d'une culture surf et de sa popularité au Québec et à Montréal, les sports de glisse se sont généralisés à plusieurs sphères et plusieurs territoires. Il est désormais courant de voir les adeptes de glisse faire de la planche à neige et du snowskate sur toutes les montagnes; les adeptes de planche à voile, de kite-surf, de wake-surf, de kayak de rivière ou de kayak de mer parcourir toutes les rivières et les plans d'eau disponibles; les adeptes de vol libre (parapentes, voile libre) écumer le ciel dans plusieurs régions.

Parmi les nouvelles pratiques sportives en milieu naturel, on retrouve quelques activités qui s'insèrent véritablement dans le paysage et qui interpellent les acteurs touristiques concernés : le snowskate, le kite-surf et le wake-surf. Le snowskate est un petit skateboard avec un mini-ski fixé sous la planche. L'usager n'est retenu par la planche que par une corde de sécurité comme pour une planche de surf classique. Le kite-surf se pratique avec une petite planche de surf tractée par un cerf-volant de performance, les vitesses pouvant être très élevées. Le wake-surf se pratique avec une petite planche de surf tractée par un bateau un peu à l'image du ski nautique. Il existe même une version alternative au surf de rivière où une embarcation motorisée génère une vague artificielle dans son sillage, permettant à un surfeur de suivre le bateau sans attaches et sans aucune forme de traction sinon pour la propulsion initiale. Contrairement au surf de rivière qui consiste à demeurer dans la vague et à faire du surplace, cette dernière pratique permet un mouvement vers l'avant.

Fait récent, le mont Royal et les parcs montréalais sont envahis par les planches. Les snowskates sont utilisés l'hiver sur la montagne et dans les cimetières limitrophes, alors que les longboards le sont tout autant pendant les autres saisons. Une compétition internationale de sports de gravité, le Top Challenge, s'est déroulée pendant quelques années sur la voie Camillien-Houde, qui traverse le mont Royal, et plusieurs compétiteurs de longboards, de luges de rue, de patins à roues alignées et autres s'y disputaient des courses à des vitesses variant entre 60 et $110 \mathrm{~km}$ à l'heure! (Filiatrault, 2006) Le surf de rivière est aussi praticable sur quelques vagues autour de l'île et on dénombre environ 500 adeptes réguliers des quelques vagues situées près du complexe résidentiel Habitat 67 et des rapides de Lachine situées au sud-ouest du territoire. À l'extrémité ouest de l'île, les plans d'eau étant beaucoup plus larges, on retrouve la planche à voile qui côtoie les pratiquants de wake-surfet de kite-surf. La Ville de Montréal, sur plusieurs tribunes médiatiques, sur des publicités de promotion touristique et parfois sur des panneaux et oriflammes localisés dans les quartiers centraux, a largement utilisé cette idée plutôt singulière à l'effet que «Montréal était une ville où il était possible de surfer! ». Dans le cadre de son programme «ça n’a pas de prix", l'entreprise Mastercard (2008) a même produit un court reportage où un adepte de la vague d'Habitat 67 proclame haut et fort que la ville «a le potentiel pour devenir le Hawaii du surf de rivière».

\section{Conclusion}

Montréal ne se distingue pas plus que d'autres villes nordaméricaines ou d'ailleurs dans le monde sur cette montée en puissance de la culture surf et de la glisse urbaine, mais elle en est un exemple édifiant. Comme le vélo et le patin à roues alignées à une certaine époque, on assiste à une réappropriation de la rue par de nouveaux usagers et cette fois, c'est la culture surf et l'esprit de la glisse qui en est le catalyseur à travers l'utilisation d'une grande diversité de planches et autres appareils de glisse.

La ville devient ainsi un territoire métissé de pratiques sportives plurielles et de tensions nouvelles, mais constructives entre usagers. Une telle diversification de pratiques sportives engendre surtout de nouveaux besoins en termes de réglementation, de codes de sécurité sur la voirie et l'espace public, mais aussi dans l'offre d'espaces, de lieux et de circuits adaptés. Les intervenants touristiques, les planificateurs urbains, aménagistes et autres gestionnaires du territoire seront de plus en plus interpellés par les nouvelles pratiques de glisse. Plusieurs autres études empiriques et analyses seront indispensables pour bien réagir à cette recomposition des modes de déplacement et des pratiques sportives. L'ensemble du phénomène est à ce point nouveau qu'il n'existe d'ailleurs que très peu de données et d'observations pertinentes pour bien évaluer son impact direct et indirect. Or, le sujet est trop fascinant et trop porteur pour être passé sous silence. Il reste assurément beaucoup de recherches complémentaires et d'études à mener pour enrichir notre réflexion.

Expression des nouveaux besoins individuels et sociaux dans l'espace urbain, mais aussi dans plusieurs autres types de territoire, le surfeur urbain, nautique ou de neige nous permet d'entrevoir une nouvelle urbanité et plus globalement, un nouveau rapport au territoire. La rue devient, par exemple, un espace où il fait bon prendre la vague asphaltée. La montagne, les plans d'eau, la neige, le ciel et tous les autres environnements deviennent des territoires à explorer, à apprivoiser et à conquérir par de nouvelles sensations de glisse, de nouveaux repères. La culture surf réactivée et renouvelée de ces dernières années a permis un décloisonnement des pratiques sportives et une valorisation des nouvelles appartenances individuelles et sociales en rapport avec ces territoires, le tout avec un souci d'harmonie et de symbiose avec l'environnement. Avec cette idée d'une refondation du rapport traditionnel du surfeur avec son environnement, les nouveaux territoires du surf dans la ville seront vraisemblablement les plus spectaculaires et les plus inusités à s’imposer. 


\section{Bibliographie}

AUGUSTIN, Jean-Pierre (1994) Surf Atlantique; Les territoires de l'Éphémère, Bordeaux, Éditions de la Maison des Sciences de l'Homme d'Aquitaine, $272 \mathrm{p}$.

ADAMKIEWICZ, Éric (2001) «Glisse urbaine et redéfinition de l'espace urbain» dans Alain Loret et Anne-Marie Waser (dir.), «Glisse urbaine; L'esprit roller : liberté, apesanteur, tolérance», Paris, Le Journal Autrement, Mutations, $\mathrm{n}^{\circ}$ 205, juin, p. 200-212.

ARTHUR, Dave (2003) «Corporate sponsorship of sport : its impact on surfing and surf culture» dans J. Skinner; K. Gilbert et A. Edwards (dir.), Some Like it Hot: The Beach as a Cultural Dimension, Oxford, Meyer and Meyer Sport, $205 \mathrm{p}$.

BESSAS, Yves (1982) La glisse, Paris, Fayard, 237 p.

BORDEN, Iain (2001) Skateboarding, Space and the City; Architecture and the Body, Oxford, Berg, 318 p.

BRADY, Kathleen (1986) «If everybody had an ocean... but even water is not essential to join the surf craze», Time, vol. 128, 18 août, p. 69.

BROWN, Matt (1997) Billion Dollar Breakers : The Professional Surfing World, traduction libre d'une émission de radio, «Radio Nation». 13 avril.

CAROL, Fred (1995) Surfers; Dompteurs de vagues, Boulogne, Sirene, $129 \mathrm{p}$.

COLBURN, Bolton; Ben Finney; Tyler Stallings; C.R. Stecyk; Deanne Stillman et Tom Wolfe (2002) Surf Culture; The Art History of Surfing, Laguna Art Museum in association with Gingko Press.

CCS (Conseil canadien du ski) (2007) Rapports des activités; développement des skieurs/surfeurs; et Industrie canadienne du ski et du surf des neiges : Faits et statistiques 2006-2007, septembre.

DE SOULTRAIT, Gibus (1995) L’homme et la vague, Guéthary, Vent de terre, $119 \mathrm{p}$.

DOLNICAR, Sara et Martin FLUKER (2003) «Behavorial Market Segments Among Surf Tourists : Investigating Past Destination Choice», Journal of Sport Tourism, Vol. 8, nº 3, p. 186-196.

FILIATRAULT, Olivier (2006) Enquêtes sur le profil des pratiquants de longboard à Montréal, 2004 et 2005, Groupe de recherche sur les espaces festifs (GREF), Département de géographie, UQAM, 54 p.

FORD, Nick et David BROWN (2006) Surfing and Social Theory; Experience, embodiment and narrative of the dream glide, Londres, Routledge, $204 \mathrm{p}$.

HAMM, Keith David (2004) Scarred for Life, San Francisco, Chronicle Books, $231 \mathrm{p}$.

HUMPHREYS, Duncan. (2003) "Selling out snowboarding: The alternative response to commercial co-optation", dans R. Rinehart et S. Sydor (dir.) To the Extreme: Alternative Sports, Inside and Out, Albany, State University of New York Press, 436 p.

KAMPION, Drew et Bruce BROWN (1998) Stoked; L'histoire du surf, Los Angeles, Evergreen, $224 \mathrm{p}$.

KARSTEN, Lia et Eva PEL (2000) «Skateboarders exploring urban public spaces : Ollies, obstacles and conflicts", Journal of Housing and the Built Environment, $\mathrm{n}^{\circ}$ 15, p. 327-340.

KING, Don et Maurice REBEIX (1997) Aloha Blue, Biarritz, Vent de Terre, $137 \mathrm{p}$.

LEFEBVRE, Sylvain et Daniel LATOUCHE (2001) «La glisse urbaine en Amérique du Nord», dans Alain Loret et Anne-Marie Waser (dir.), Glisse urbaine; L'esprit roller : liberté, apesanteur, tolérance, Paris, Le Journal Autrement, Mutations, nº 205, juin, p. 188-199.

LEFEBVRE, Sylvain et Jean-Marc CHOUINARD (2003) «Le patin à roues alignées à Montréal; Expression, accommodement ou revendication», dans Sylvain Lefebvre (dir.), Sports et villes; enjeux économiques et socioculturels, Collection Géographie contemporaine, Québec, Presses de l'Université du Québec, 241 p.

LEFEBVRE, Sylvain (2004) «Surf nouvelle vague; L'arrivée des longboards vient encore compliquer le partage de la voie publique» La Presse, 28 novembre, p. A9.

LHOTE, Gilles (2003) Surf Attitudes, Neuilly-sur-Seine, Michel Lafon, 192p.

LORET, Alain (1995) Génération glisse, Paris, Autrement, 325 p.

LORET, Alain et Anne-Marie WASER (dir.) (juin 2001) «Glisse urbaine; L'esprit roller : liberté, apesanteur, tolérance», Paris, Le Journal Autrement, Mutations, $\mathrm{n}^{\circ}$ 205, $260 \mathrm{p}$.

MasterCard (2008) "Ça n'a pas de prix», MasterCard Canada Inc., <www. canapasdeprix.ca> (Consulté le 23 juin 2008)

NSSRA (National Ski and Snowboarding Retailers Association) (2001) Snowboarding Participation 1993-2000, Snowboarding Association, Mt. Prospect Il.

ODIT (Observation, Développement et Ingénierie Touristiques) Agence publique de développement et d'ingénierie touristique (2009), Les chiffres-clés du tourisme de montagne en France, Paris.

PÉGARD, Olivier (1998) «Une pratique ludique urbaine : Le skateboard sur la Place Vauquelin à Montréal ", Cahiers internationaux de Sociologie, Vol. CIV, p. 185-202.

POIRIER, Jean-Étienne (2000) Hopupu; Les délices de la glisse, Sillery, Septentrion, $182 \mathrm{p}$.

POIZAT-NEWCOMB, Steve (1999) «The Genesis of a Sports Tourism Activity-Surfing", Journal of Sport \& Tourism, vol. 5, n 4, p. 5-25.

SAYEUX, Anne-Sophie (2008) Surfeurs, l'être au monde; Une analyse anthropologique, Rennes, Presses Universitaires de Rennes, 217 p.

SGMA International (2006) Sports Participation in America; Skateboarding, 2005 Edition, American sports Data Inc, 53 p.

VIVONI, Francisco (2009) "Spots of Spatial Desire; Skateparks, Skateplazas, and Urban Politics”, Journal of Sport \& Social Issues, vol. $33, \mathrm{n}^{\circ}$ 2, mai, p. 130-149.

WHEATON, Belinda (dir.) (2004) Understanding Lifestyle Sport; Consumption, Identity and Difference, Londres, Routledge, $220 \mathrm{p}$.

WIELAND, Flavio (1999) Les nouvelles pratiques sportives en milieu urbain: La rue comme espace de jeu. Le cas du skateboard à Montréal, Mémoire de maitrise, sous la direction de S. Lefebvre et J.-B Racine, GREFUQAM, 125 p. 